\title{
Description of non-polio enteroviruses identified in two national surveillance programmes in South Africa
}

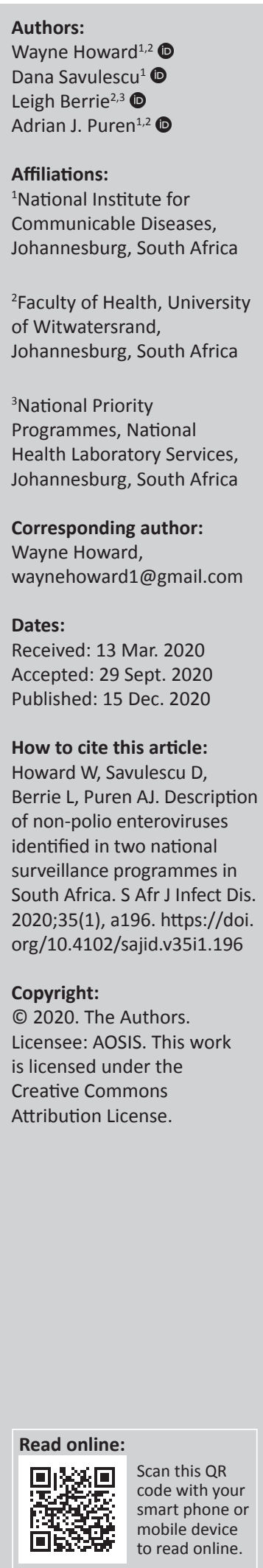

Background: Human enteroviruses (EV) consist of 106 serotypes and four species: EV-A, EV-B, EV-C and EV-D. Enteroviruses cause clinical symptoms varying from severe to mild. Knowledge of EV burden in South Africa is limited, and as non-polio EV are important causes of acute flaccid paralysis (AFP) and meningitis, information on the circulating serotypes is vital.

Methods: Between 2010 and 2012, a total of 832 stool and viral isolate specimens were obtained from two national surveillance programmes at the National Institute for Communicable Diseases: the Rotavirus Sentinel Surveillance Programme (RSSP) and the AFP surveillance programme. Real-time polymerase chain reaction and Sanger sequencing were performed to detect and serotype EV.

Results: Non-polio EV were detected in 446 specimens, of which 308 were sequenced. Stool specimens yielded a greater variety of serotypes than viral cultures. EV-B viruses were predominant $(58.44 \%$ ), whilst EV-C viruses were detected in $31 \%$ of the specimens tested. South African prevalence for these viruses was higher than other countries, such as France with less than $2 \%$, and Spain and the United States with less than $10 \%$. The most common serotype detected was Enterovirus 99 (EV-C, 8.63\%), which has not been reported in other regions.

Conclusion: Direct sequencing from stool specimens yields a broader, more comprehensive description of EV infections compared to sequencing from viral cultures. Disease-associated serotypes were detected, but only in small numbers. This study provides a baseline for EV strain circulation; however, surveillance needs to be expanded to improve EV knowledge in South Africa.

Keywords: human enteroviruses; meningitis; NICD; stool specimens; disease-associated serotypes; South Africa.

\section{Introduction}

Human enteroviruses (EV), a part of the Picornaviridae family in the Enterovirus genus, ${ }^{1}$ are divided into four species: EV-A, EV-B, EV-C and EV-D. ${ }^{2}$ Although EV infections occur in early childhood, they may also occur in later years because of the high number of EV serotypes. ${ }^{3}$ Despite most infections being asymptomatic, large numbers of symptomatic infections are estimated to occur every year, contributing to morbidity and mortality. ${ }^{3}$ Most EV infections are mild, causing headaches, rhinitis and rash; however, some infections can lead to serious diseases such as cardio myelitis, flaccid paralysis and diarrhoea, particularly in infants ${ }^{4}$ and the immunocompromised. ${ }^{5}$ Enteroviruses are the leading cause of viral aseptic meningitis, ${ }^{6}$ and are also implicated in a wide range of acute and chronic infections ranging from non-febrile disease, conjunctivitis and upper respiratory infections, to hand-foot-and-mouth disease. ${ }^{6}$

Enterovirus incidence is difficult to determine as transmission can be endemic, sporadic or epidemic. Additionally, many infections are subclinical. ${ }^{2}$ Some EV cause multiple syndromes that may develop into serious complications from asymptomatic or subclinical infections. ${ }^{6}$ Large populations often have multiple serotypes circulating concurrently. Outbreaks can occur with varying degrees of severity and are generally characterised by serotype, time, location and disease. ${ }^{7,8}$

Most studies on EV in South Africa have focused on polioviruses (PV) or outbreaks of non-polio EV. ${ }^{9,10,11,12,13}$ Polioviruses have caused significant outbreaks of acute flaccid paralysis (AFP) in the past and have been the focus of vaccine programmes, with the aim of eradication, since $1988 .{ }^{14}$ Acute flaccid paralysis is caused by PV infection of spine nerve cells, leading to irreversible 
damage that results in permanent paralysis or death. ${ }^{5}$ Studies investigating EV outbreaks in South Africa have been short term, looking at one particular virus-associated disease. ${ }^{12,13}$ An earlier retrospective study on a meningitis outbreak described the prevalence of non-polio EV in Cape Town in 1993. ${ }^{11}$ A recent study from South Africa examined the prevalence of EV in respiratory disease patients from 2009 to 2014, using respiratory swabs and lavages. ${ }^{9,10}$ These studies give an incomplete view on EV prevalence and have limited use in outbreak control or disease surveillance. Typing EV has evolved with the development of molecular analysis technology. Polymerase chain reaction (PCR) and genetic sequencing are now used to genotype $\mathrm{EV}$ into the conventionally assigned species and serotypes. ${ }^{15}$

Detection and elimination of PV has been achieved through the AFP surveillance network, which surveys all AFP cases detected, including those where polio is not the cause. ${ }^{14}$ Poliovirus was eliminated from South Africa in 1989 and thus the investigation of AFP cases remains essential because other non-polio EV may be causative organisms. ${ }^{16,17}$ The circulation and changes in predominance of EV serotypes are complex, and surveillance programmes may aid in tracking and identifying EV serotypes. ${ }^{18}$ Two routine surveillance systems in South Africa - the AFP surveillance network and the Rotavirus Sentinel Surveillance Programme (RSSP) provide potential specimens to investigate circulating EV. Specimens from these two programmes may enable detection of EV potentially causing AFP, as well as those causing diarrhoea or gastroenteritis.

The AFP surveillance network collects stool specimens from children under the age of 15 with AFP, or adults with AFP where polio is suspected. The RSSP collects stool specimens from patients under the age of 5 admitted to hospital for diarrhoea to determine the effectiveness of the rotavirus vaccine introduced in 2009. ${ }^{19}$

Whilst this study sought to obtain detailed information on EV circulation in South Africa, the specimen type was limited. Cerebrospinal fluid (CSF), conjunctivitis swabs, rash vesicle fluid and respiratory and stool specimens should be surveyed to establish a complete picture of EV circulation and disease burden. ${ }^{20}$

This study aimed to determine the epidemiology of non-polio EV circulating in South Africa from 2010 to 2012. We investigated any serotype-disease association in stool specimens obtained from AFP suspected infections, which may give an indication of EV associated with neurological infections; and from patients with diarrhoea, elucidating EV involvement in enteric diseases and the expansion of EV surveillance.

\section{Materials and methods}

\section{Specimen sources}

Eight hundred and thirty-two stool and viral isolate specimens, collected between January 2010 and December 2012, were sourced from the AFP surveillance programme and the RSSP at the National Institute for Communicable Diseases (NICD), Johannesburg, South Africa.

Specimens from the AFP surveillance programme were selected by obtaining all the positive non-polio EV viral isolates determined through EV-like cytopathic effect (CPE) ${ }^{21}$ One stool specimen that showed no CPE on cell culture from each of the nine provinces in South Africa each month was also included for direct detection. A total of 175 non-polio EV-positive cultures were collected between 2010 and 2012, and a further 95 culture-negative stools were obtained from January to December 2012. Specimens were discarded on an annual basis and thus no raw stools were available for 2010 and 2011.

The RSSP supplied 562 stool specimens from four provinces Gauteng, Kwa-Zulu Natal, Western Cape and Mpumalanga covering a mixture of rural, peri-urban and urban populations. ${ }^{19}$ The hypothesised percentage frequency was based on the number of non-polio EV detected in the AFP surveillance network for South African patients per year. We selected the first four specimens arriving at the NICD from each site per month for the years 2010-2012 calculated by Equation 1:

$n=\left[\mathrm{Z}^{2 *} \mathrm{p} *(1-\mathrm{p})\right] / \mathrm{c}^{2}$

[Eqn 1]

- $n=$ sample size

- $p=$ hypothesised percentage frequency of outcome $(15 \%)$, confidence level of $95 \%$

- $c=0.05$

- $Z=1.96$

The Western Cape started collecting specimens in May 2010, resulting in a lower total specimen number for 2010 (175 specimens) from that site.

\section{Specimen preparation}

Viral Ribonucleic Acid (RNA) extractions were conducted on culture samples and stool samples using the automated Maxwell 16 system (Promega, Madison, Wisconsin, United States), or manually using the Qiagen Qiamp Viral Mini Kit (Qiagen, Venlo, Netherlands). For stool samples, both manual and automated extractions were preceded by stool dilution in stool transport and recovery (STAR) buffer (Roche, Mannheim, Germany), to ensure adequate removal of (PCR) inhibitors. The treated stool specimens were centrifuged at $1500 \mathrm{~g}$ for $1 \mathrm{~min}$ at room temperature to sediment the solids, with the supernatant aliquoted. Specimens that failed to yield a useable nucleotide sequence were processed manually and re-sequenced.

\section{Polymerase chain reaction and sequencing}

The real-time PCR protocol from Nijhuis et al..$^{22}$ was used to screen specimens for the presence of $\mathrm{EV}$, followed by amplification and sequencing of EV-positive specimens using a semi-nested assay and degenerate PCR primers, sequencing primers and protocols designed by Nix et al. ${ }^{23}$ 
Sanger sequencing was conducted as per the BigDye Terminator (version 3.1) Cycle Sequencing Kit (Life Technologies, Carlsbad, California, United States) and analysed on the ABI 3130 genetic analyser (Life Technologies, Carlsbad, California, United States).

Specimens were serotyped using Oberste's criteria for EV typing, that is, greater than $75 \%$ nucleotide sequence homology to the published sequences. ${ }^{15}$ The National Centre for Biotechnology Information (NCBI) database was utilised to compare the EV sequences obtained in the study using the BLAST (Basic Local Alignment Search Tool) function.

\section{Statistics}

The gender prevalence and median age of the selected cases were calculated from surveillance data available, with the interquartile range determined by subtracting the lower quartile from the upper quartile. One-way Analysis of Variance (ANOVA) and T-tests were done on GraphPad Prism (University of Leicester, United Kingdom). A $p$ value of 0.05 and lower was considered statistically significant.

\section{Ethical consideration}

Ethics approval was obtained from the University of Witwatersrand Ethics Committee (M120467, M119034 and M111145).

\section{Results}

The EV PCR screen was conducted on 832 specimens with $446(53.61 \%)$ specimens reported positive for EV. Male patients constituted $55.51 \%(246 / 446)$ of the EV-positive cases; most specimens came from children under the age of 5, with a median age of 1 year and interquartile range of 1.48 . Patients under the age of 1 made up 49.33\% (220/446) of the positive specimens, $41.26 \%(184 / 446)$ were between the ages of 1 and 5 years and the remainder $(7.62 \% ; 34 / 446)$ over the age of 5 (Table 1). The unaccounted for ages $(1.79 \%(8 / 446)$ and gender $(2.47 \% 11 / 446)$ are unknown.

Sixty-three serotypes were detected from three species groups, EV-A, EV-B and EV-C. No EV-D serotypes were identified. EV-A, EV-B and EV-C were detected in $10.7 \%$ $(33 / 308), 58.4 \%(180 / 308)$ and $30.8 \%(95 / 308)$ of specimens, respectively. In EV-A, there were 12 serotypes identified, with CVA5 most frequently detected (6/32 detections, $18.2 \%$ ). Thirty-seven EV-B serotypes were detected with the most common serotype being CVB3 (in 15/180 [8.3\%] specimens), followed by Ec6 (14/180 specimens, 7.8\%). EV-C had 14

\begin{tabular}{lc}
\multicolumn{2}{l}{ TABLE 1: Gender and age of patients with enterovirus-positive specimens. } \\
\hline Variable & $\%$ \\
\hline Gender & \\
Male & 55.16 \\
Female & 42.38 \\
Patients' age $\dagger$ & \\
$<1$ year old & 49.33 \\
$>1$ year and < 5 years old & 41.26 \\
$>5$ years old & 7.62 \\
\hline
\end{tabular}

$\dagger, 90.59 \%$ under 5 years old.
TABLE 2: Total number of serotypes detected.

\begin{tabular}{|c|c|c|c|}
\hline Species & Serotype & $\begin{array}{c}\text { Overall } \\
\text { detections }\end{array}$ & $\begin{array}{l}\text { Individual percentage } \\
\text { of total detections }\end{array}$ \\
\hline \multirow[t]{12}{*}{ EV-A $\dagger$} & CA2 & 4 & 1.30 \\
\hline & CA4 & 1 & 0.32 \\
\hline & CA5 & 6 & 1.95 \\
\hline & CA6 & 3 & 0.97 \\
\hline & CA7 & 3 & 0.97 \\
\hline & CA8 & 1 & 0.32 \\
\hline & CA10 & 4 & 1.30 \\
\hline & CA14 & 2 & 0.65 \\
\hline & CA16 & 3 & 0.97 \\
\hline & EV71 & 2 & 0.65 \\
\hline & EV76 & 1 & 0.32 \\
\hline & EV114 & 3 & 0.97 \\
\hline \multirow[t]{37}{*}{ EV-B } & CB1 & 5 & 1.62 \\
\hline & $\mathrm{CB} 2$ & 4 & 1.30 \\
\hline & CB3 & 15 & 4.87 \\
\hline & CB4 & 4 & 1.30 \\
\hline & CB5 & 8 & 2.60 \\
\hline & CB6 & 1 & 0.32 \\
\hline & CA9 & 3 & 0.97 \\
\hline & Ec1 & 3 & 0.97 \\
\hline & Ec2 & 1 & 0.32 \\
\hline & Ec3 & 8 & 2.60 \\
\hline & Ec4 & 2 & 0.65 \\
\hline & Ec5 & 1 & 0.32 \\
\hline & Ec6 & 14 & 4.55 \\
\hline & Ec7 & 6 & 1.95 \\
\hline & Ec9 & 7 & 2.27 \\
\hline & Ec11 & 10 & 3.25 \\
\hline & Ec12 & 5 & 1.62 \\
\hline & Ec13 & 11 & 3.57 \\
\hline & Ec14 & 7 & 2.27 \\
\hline & Ec15 & 4 & 1.30 \\
\hline & Ec16 & 3 & 0.97 \\
\hline & Ec17 & 1 & 0.32 \\
\hline & Ec18 & 1 & 0.32 \\
\hline & Ec19 & 9 & 2.92 \\
\hline & Ec20 & 6 & 1.95 \\
\hline & Ec21 & 7 & 2.27 \\
\hline & Ec24 & 6 & 1.95 \\
\hline & Ec25 & 7 & 2.27 \\
\hline & Ec27 & 4 & 1.30 \\
\hline & Ec29 & 4 & 1.30 \\
\hline & Ec30 & 5 & 1.62 \\
\hline & Ec31 & 1 & 0.32 \\
\hline & Ec32 & 1 & 0.32 \\
\hline & EV75 & 2 & 0.65 \\
\hline & EV77 & 1 & 0.32 \\
\hline & EV80 & 2 & 0.65 \\
\hline & EV88 & 1 & 0.32 \\
\hline \multirow[t]{14}{*}{ EV-C $\S$} & CA1 & 2 & 0.65 \\
\hline & CA11 & 4 & 1.30 \\
\hline & CA13 & 11 & 3.57 \\
\hline & CA17 & 4 & 1.30 \\
\hline & CA19 & 3 & 0.97 \\
\hline & CA20 & 2 & 0.65 \\
\hline & CA21 & 2 & 0.65 \\
\hline & CA22 & 4 & 1.30 \\
\hline & CA24 & 16 & 5.19 \\
\hline & EV99 & 27 & 8.77 \\
\hline & EV102 & 1 & 0.32 \\
\hline & PV1-SABIN & 2 & 0.65 \\
\hline & PV2-SABIN & 5 & 1.62 \\
\hline & PV3-SABIN & 12 & 3.90 \\
\hline Total & - & 308 & - \\
\hline
\end{tabular}

$\dagger$, Species group EV-A $(\%)=10.71 ; \ddagger$, Species group EV-B $(\%)=58.44 ; \S$, Species group $\mathrm{EV}-\mathrm{C}(\%)=30.84$. 
serotypes, where EV99 was detected in 27 of 95 specimens $(28.4 \%)$ and CVA24 in 16 of 95 specimens (16.8\%) (Table 2).

The AFP surveillance programme yielded 168 of the $270(62.22 \%)$ EV-positive cases from the culture and stool specimens, of which $147(87.5 \%, n=168)$ were serotyped. The species groups detected included EV-A (7.5\%; 11/147), EV-B (79.6\%; 117/147) and EV-C (12.9\%; 19/147). The most common serotype was CVB3, followed by Ec6. Enterovirus 99 was detected in seven specimens: three from viral culture positive cases, and four from AFP stool specimens.

From the RSSP subset, $49.11 \%$ (276/562) were positive for EV. Serotypes were identified from $58.3 \%(161 / 276)$ of stools including species group EV-A (13.7\%; 22/161), EV-B (39.1\%; $63 / 161)$ and EV-C (47.2\%; 76/161). Enterovirus 99 was the most frequent serotype detected $(12.4 \% ; 20 / 161)$, followed by CVA24 $(8.7 \% ; 14 / 161)$.

Although EV-B was the predominant group detected overall, EV99 from the EV-C group was the serotype most frequently detected overall $(8.8 \% ; 27 / 308)$, followed in decreasing frequency by CVA24 (5.2\%, EV-C; 16/308), CVB3 (4.87\%, EV-B; 15/308) and Ec6 (4.55\%, EV-B; 14/308). The serotypes detected from EV-A, EV-B and EV-C groups were heterogeneous and there was no dominant EV-A and EV-B serotype. EV-A and EV-B serotypes did not show an infection bias towards gender, although EV-C infected more males (61/94 detections, $64.89 \%)$ than females (33/94 detections, $35.11 \%$ ) (Figure 1). This was not statistically significant $(p$-value $=1.00$, confidence interval $[\mathrm{CI}]: 95 \%)$.

Specimen type influenced the groups and serotypes identified. Many of the AFP programme specimens yielded EV-B (90.4\%; 113/125) strains. Two serotypes were most frequently detected: CVB3 in 13 specimens and Ec6 in 12 specimens. The stool specimens yielded mainly EV-C serotypes: $63.6 \%(14 / 22)$ from the culture-negative, AFP stool specimens; and $47.2 \%(76 / 161)$ from the RSSP specimens. Enterovirus 99 was the most frequently detected serotype in the combined sets of stool specimens. Enteroviruses CVA1, 5,
8, 9, 10, 11, 13, 15, 17, 24, and Ec15, were serotypes detected in AFP stool specimens, but not from cell culture.

The distribution of EV serotypes was varied and widespread across the country and no distinct distribution pattern in the different provinces was observed. Most specimens were collected from the provinces that included the RSSP collection sites as well as from the AFP surveillance programme: Western Cape, Gauteng, Kwa-Zulu Natal and Mpumalanga (Figure 2).

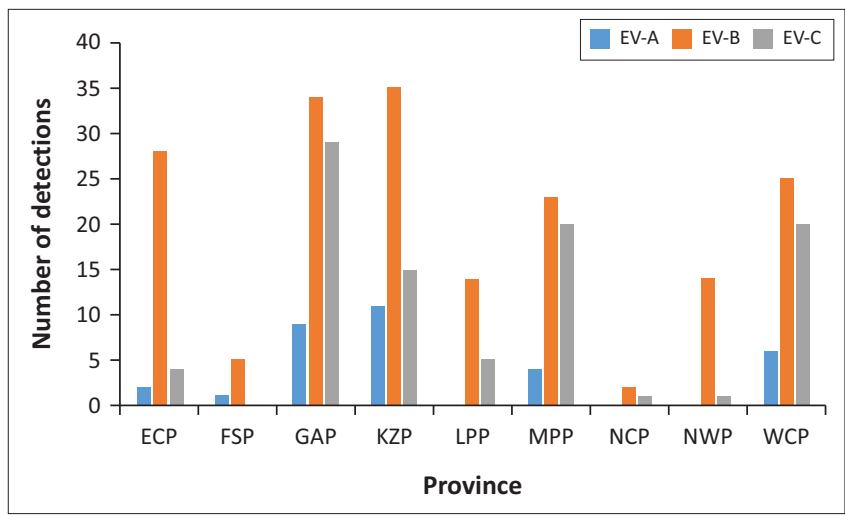

ECP, Eastern Cape; FSP, Free State; GAP, Gauteng; KZP, Kwa-Zulu Natal; LPP, Limpopo; MPP, Mpumalanga; NCP, Northern Cape; NWP, North West; WCP, Western Cape.

FIGURE 2: Distribution of EV-A, EV-B and EV-C per province in South Africa.

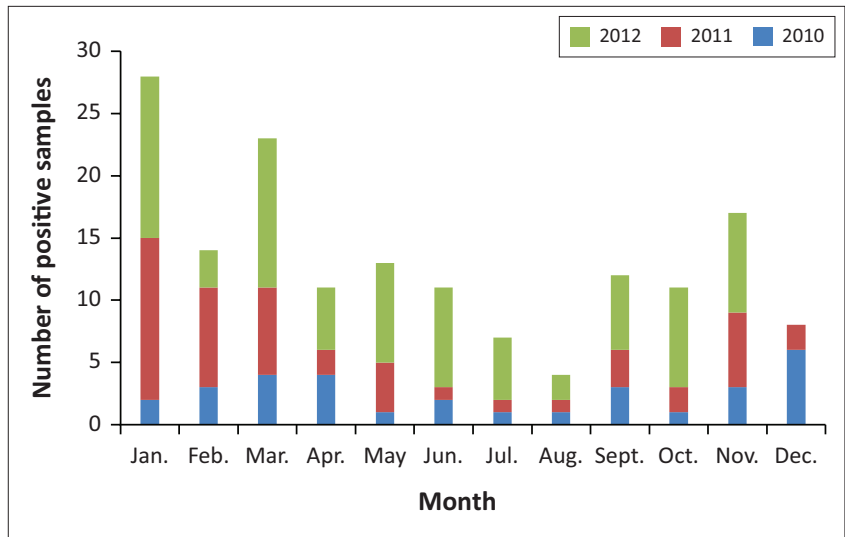

FIGURE 3: Total number of positive specimens in the acute flaccid paralysis surveillance programme by month, for 2010-2012.

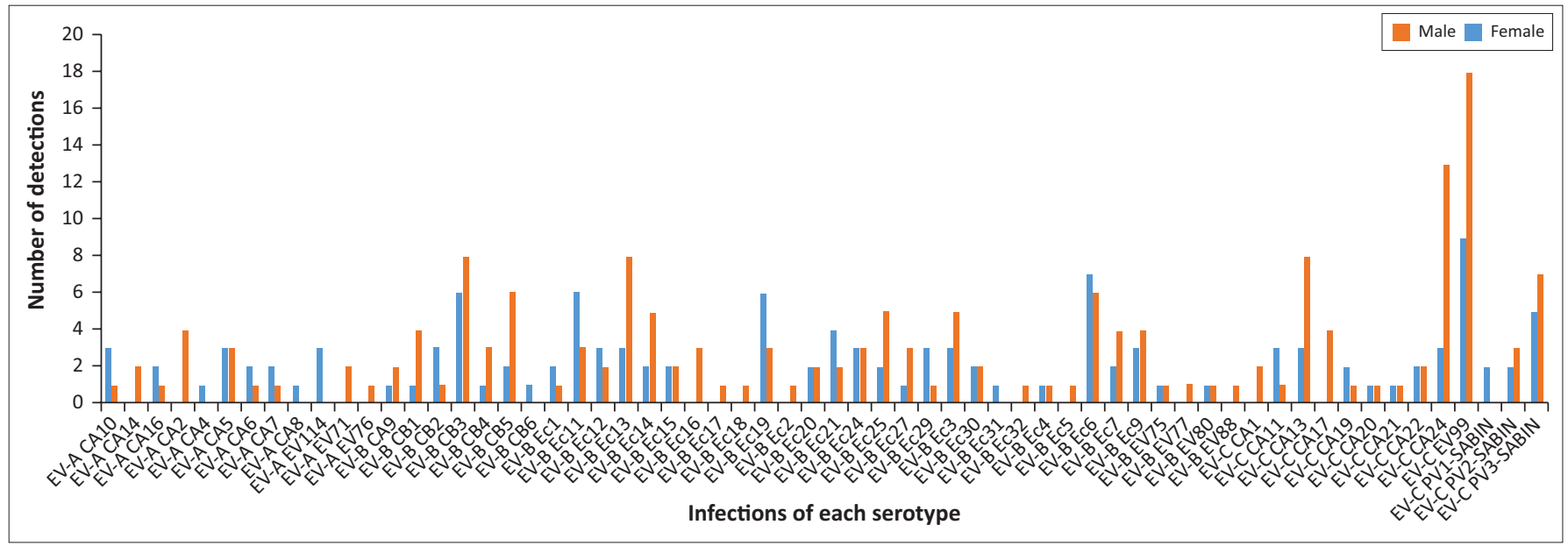

FIGURE 1: Infections of each serotype in males and females. 
There was no clear seasonality in the distribution of the positive EV specimens detected in the RSSP. In the case of the AFP surveillance, seasonality was observed with January to March showing a peak in infections (Figure 3), although this was not statistically significant ( $p$-value $=0.4433$; CI: 95\%).

\section{Discussion}

No predominant EV serotype was detected, and a wide distribution of serotypes across EV-A, EV-B and EV-C was observed. Serotype distribution followed for the most part gender and age patterns seen historically across the world. 2,3,5,6 Some known disease-associated serotypes were detected but were not more prevalent than other serotypes.

The AFP surveillance programme obtained specimens from all nine provinces, which allowed for description of a countrywide EV distribution over a 3-year period. The use of this programme was advantageous as the specimens originated from all provinces and most districts in the country, and the infrastructure for specimen collection and transport had already been established.

The RSSP ${ }^{19}$ sites covered different regions in South Africa and overlapped areas covered by the AFP surveillance programme. The sites were in the Gauteng, Western Cape, Kwa-Zulu Natal and Mpumalanga provinces. ${ }^{24}$ Using the national surveillance systems in place ensured good population coverage and direct stool specimen screening, without a virus isolation intermediate step. This allowed for detection of serotypes that are impossible or difficult to grow in cell cultures.

The pattern of EV distribution seen in the cell culture specimens was consistent with other studies worldwide, $25,26,27,28,29,30,31$ with EV-B being the predominant group and EV-D being the least prevalent group. However, the stool specimens screened in our study yielded additional EV-C viruses $(47.20 \%$ in the RSSP specimens and $63.64 \%$ in the AFP stool specimens). Our data show that using only cell culture for EV identification may limit and bias the results towards isolating EV-B viruses.

Although EV-B was the most prevalent species detected, EV99 (EV-C) was the most prevalent serotype detected over 3 years -27 out of 308 specimens typed $(8.63 \%)$. Within each year, EV99 was the most prevalent serotype found along with Ec6 (9 viruses, 2010), CVB5 and Ec14 (6 viruses each, 2011) and Ec13 (10 viruses, 2012). This finding was unusual, as EV99 has not been previously detected as a common serotype in other studies in China and Finland. ${ }^{32,33}$

Despite many serotypes detected in our study being associated with disease, these serotypes did not contribute significantly to the total number of viruses detected. Strain EV71 (isolated from the AFP culture positive specimens) has been associated with aseptic meningitis, ${ }^{34,35}$ and although its presence in patients presenting with neurological symptoms is expected, only two cases were detected over the three surveillance period. The more recently classified EV, namely,
EV80, EV88, EV102 and EV114, were also detected in this study. These viruses are rarely detected and/or newly discovered and have no clear disease association.

The distribution of the serotypes across the country did not show any distinct pattern, although many more types were detected in Gauteng, Mpumalanga, Kwa-Zulu Natal and Western Cape. This is likely because of the larger numbers of specimens obtained from these provinces, as well as their mixed populations. Further studies, with specimens collected more evenly between the nine provinces, will be required in order to confirm this. Ideally, a surveillance programme tailored to detect EV symptoms including handfoot-and-mouth disease, aseptic meningitis, myocarditis and respiratory disease would be required for improved detection. ${ }^{9,10,18,36}$

Serotype distribution varies over time within a geographical location, ${ }^{37}$ as well as over large distances, such as between continents. Enterovirus Ec30 is the predominant serotype in Europe, ${ }^{38}$ whilst EV71 is the predominant serotype worldwide. ${ }^{39}$ In our study, EV99 was found to be the most common serotype in South Africa. Other studies ${ }^{32,33}$ have not definitively linked EV99 to a disease, and with the virus still relatively unknown, further investigations are required to discover any clinical relevance. The higher levels of EV99 detected compared to other countries may be because of lack of serotyping studies, as well as the use of stool specimens in this study instead of viral isolates. ${ }^{7,840,41}$ Enterovirus 99 does not grow in cell cultures as readily as viruses from EV-B. Detection of EV directly from stool specimens allows for a more accurate distribution of EV within a population. This genotyping method is faster than virus isolation and so results from an outbreak can be utilised with epidemiological information to prevent further transmission. ${ }^{23}$

Hellferscee and colleagues ${ }^{9,10}$ published two studies that showed EV strains in respiratory patients from 2009 to 2014 in South Africa, with a wide variety of serotypes detected. Their results correlate with our findings, although we did not detect EV68, more common in respiratory specimens. As EV68 has been shown to be associated with respiratory disease, this is not unexpected. ${ }^{42,43}$ All serotypes detected by Hellferscee et al., except CVA3 and EV68, were detected in our study.

This study provides a baseline for EV strain circulation and epidemiology in South Africa. With polio on the brink of eradication, other causes of AFP need to be investigated. Enteroviruses are a potential cause of these symptoms ${ }^{39}$, and this study supplies a baseline for determining which EV are circulating in the South African population. Whilst EV71, a meningitis-associated virus, and various other echoviruses were detected in this study, routine and outbreak surveillance will determine the clinical importance of these serotypes in the South African population. Inclusion of a surveillance programme would assist in detecting EV outbreaks, and utilisation of various specimen types would ensure that EV 
that replicate in different organs (e.g. EV68 found in respiratory lavages and/or swabs) were not missed.

\section{Limitations}

Limitations of this study include no control group of healthy individuals for comparison, although a study in the Philippines shows no difference between diseased and asymptomatic groups. ${ }^{44}$ The differences in serotype distribution between the surveillance programmes may have been because of the specimen type. The less severe symptom types associated with EV are not currently covered by any surveillance group in South Africa.

The current surveillance programmes in South Africa used to collect specimens target specific age groups (mostly children under 5 or 15) and are passive systems; consequently, specimen collection is only triggered when syndromes are detected. The AFP surveillance programme met all surveillance targets for the number of specimens collected in all provinces except the Northern Cape. This may underrepresent the number of viruses typed in this province.

Only one type of specimen, namely faeces, was collected from the surveillance programmes in this study, making it more difficult to obtain results (PCR inhibitors are difficult to remove and there is a risk of mixed $\mathrm{EV}$ infections).

Enterovirus genotyping has become more complex with the discovery of more serotypes. A small fragment of the VP1 gene was used for typing the EV, which was sufficient for basic typing differentiation, but more in-depth genetic analysis is required for a comprehensive description of EV in South Africa.

\section{Conclusion}

The epidemiology of EV in South Africa showed a general concordance with other studies ${ }^{16,25,29}$, and the study provided a baseline of circulating EV strains. In South Africa, various serotypes were shown to co-circulate, although EV99 was the most common virus throughout the 2010-2012 period. Strains CVA24 and EV99 accounted for 14\% of all viruses detected over the 3 years and the predominance may be explained by the natural continental differences in serotype circulation.

Specimen type influences the ability to detect different serotypes, and disease presentation affects the serotypes observed. Future surveillance may assist in determining how serotype affects disease burden. Unlike cell culture, an assay that will detect EV directly from the specimen may give a more comprehensive idea of EV strain circulation and epidemiology.

A dedicated EV surveillance programme would provide a more accurate idea of the EV disease burden on symptoms such as AFP, meningitis and encephalitis. This would be useful for outbreak detection and virological investigation. ${ }^{36}$ The development of a new vaccine to lessen the disease burden of serotypes with the association of serious effects on patients may be a by-product of the knowledge gained from EV surveillance, as predominant serotypes can be investigated as vaccine candidates.

\section{Acknowledgements}

The Poliovirus Research Foundation, grant number 12/21, funded this study. The study utilised residual specimens from the RSSP, funded by GlaxoSmithKline (E-Track 200238). However, GlaxoSmithKline were not involved in the study design, writing or publication of the study.

\section{Competing interests}

The authors have declared that no competing interests exist.

\section{Authors' contributions}

A. Puren was the project leader. L. Berrie, A. Puren and W. Howard were involved with project design. W. Howard conducted the laboratory experiments and result analysis. A. Puren, L. Berrie, D. Savulescu and W. Howard were involved in the manuscript writing.

\section{Funding information}

Funding was received from the Polio Research Foundation and the National Health Laboratory Services.

\section{Data availability statement}

Data are available upon request to the corresponding author.

\section{Disclaimer}

The views and opinions expressed in this article are those of the authors and do not necessarily reflect the official policy or position of any affiliated agency of the authors.

\section{References}

1. International Committee on Taxonomy of Viruses [homepage on the Internet] [cited 2020 Feb 03]. Available from: https://talk.ictvonline.org/

2. Pons-Salort M, Parker EP, Grassly NC. The epidemiology of non-polio enteroviruses: Recent advances and outstanding questions. Curr Opin Infect Dis. 2015;28(5): 479-487. https://doi.org/10.1097/QCO.0000000000000187

3. Fields BN. Fields' virology. 5th ed. David Mahan Knipe PMH, editor. Philadelphia, PA: Lippincott Williams \& Wilkins; 2007, p. 3177.

4. Nasri D, Bouslama L, Pillet S, Bourlet T, Aouni M, Pozzetto B. Basic rationale, current methods and future directions for molecular typing of human enterovirus. Expert Rev Mol Diagn. 2007;7(4):419-434. https://doi.org/10.1586/14737159.7.4.419

5. Rhoades RE, Tabor-Godwin JM, Tsueng G, Feuer R. Enterovirus infections of the central nervous system. Virology. 2011;411(2):288-305. https://doi.org/10.1016/ j.virol.2010.12.014

6. Tapparel C, Siegrist F, Petty TJ, Kaiser L. Picornavirus and enterovirus diversity with associated human diseases. Infect Genet Evol. 2013;14:282-293. https://doi. org/10.1016/j.meegid.2012.10.016

7. Moore M. Centers for Disease Control. Enteroviral disease in the United States, 1970-1979. J Infect Dis. 1982;146(1):103-108. https://doi.org/10.1093/infdis/ 146.1.103

8. Kumar VS, Budur SV, Odappa GH, Bankolli SY, Rao AP. Clinical profile of hand, foot, and mouth disease and its associated complications among children in Shimoga City, southern Karnataka: A hospital-based study. Indian J Public Health. 2015;59(2):141-144. https://doi.org/10.4103/0019-557X.157536

9. Hellferscee $O$, Tempia $S$, Walaza $S$, et al. Enterovirus genotypes among patients with severe acute respiratory illness, influenza-like illness, and asymptomatic individuals in South Africa, 2012-2014. J Med Virol. 2017;89(10):1759-1767. https://doi.org/10.1002/jmv.24869 
10. Hellferscee O, Treurnicht FK, Tempia S, et al. Enterovirus D68 and other enterovirus serotypes identified in South African patients with severe acute respiratory illness, 2009-2011. Influenza Other Respir Viruses. 2017;11(3):211-219. https:// doi.org/10.1111/irv.12444

11. McIntyre JP, Keen GA. Laboratory surveillance of viral meningitis by examination of cerebrospinal fluid in Cape Town, 1981-9. Epidemiol Infect. 1993; 111(2):357-371. https://doi.org/10.1017/S095026880005706X

12. Schoub BD, Johnson S, McAnerney JM, Dos Santos IL, Klaassen KI. Epidemic Coxsackie B virus infection in Johannesburg, South Africa. J Hyg (Lond). 1985;95(2):447-455. https://doi.org/10.1017/S0022172400062872

13. Yeats J, Smuts $\mathrm{H}$, Serfontein CJ, Kannemeyer J. Investigation into a school enterovirus outbreak using PCR detection and serotype identification based on the 5' non-coding region. Epidemiol Infect. 2005;133(6):1123-1130. https://doi. org/10.1017/S0950268805004462

14. Global Polio Eradication Initative [homepage on the internet]. [cited 2020 Feb 03]. Available from https://www.polioeradication.org.

15. Oberste MS, Maher K, Williams AJ, et al. Species-specific RT-PCR amplification of human enteroviruses: A tool for rapid species identification of uncharacterized enteroviruses. J Gen Virol. 2006;87(Pt 1):119-128. https://doi.org/10.1099/ vir.0.81179-0

16. Dhole TN, Ayyagari A, Chowdhary R, et al. Non-polio enteroviruses in acute flaccid paralysis children of India: Vital assessment before polio eradication. J Paediatr Child Health. 2009;45(7-8):409-413. https://doi.org/10.1111/j.1440-1754.2009. 01529.x

17. Mehrabi Z, Shahmahmoodi S, Eshraghian MR, et al. Molecular detection of different types of non-polio enteroviruses in acute flaccid paralysis cases and healthy children, a pilot study. J Clin Virol. 2011;50(2):181-182. https://doi. org/10.1016/j.jcv.2010.10.006

18. Li W, Zhang X, Chen X, et al. Epidemiology of childhood enterovirus infections in Hangzhou, China. Virol J. 2015;12:58. https://doi.org/10.1186/s12985-0150294-4

19. Msimang VM, Page N, Groome MJ, et al. Impact of rotavirus vaccine on childhood diarrheal hospitalization after introduction into the South African public immunization program. Pediatr Infect Dis J. 2013;32(12):1359-1364. https://doi. org/10.1097/INF.0b013e3182a72fc0

20. Harvala H, Broberg $E$, Benschop $K$, et al. Recommendations for enterovirus diagnostics and characterisation within and beyond Europe. J Clin Virol. 2018;101:11-17. https://doi.org/10.1016/j.jcv.2018.01.008

21. World Health Organization. Manual for the virological investigation of poliomyelitis. 4th ed. Geneva: WHO; 2004.

22. Nijhuis M, Van Maarseveen N, Schuurman R, et al. Rapid and sensitive routine detection of all members of the genus enterovirus in different clinical specimens by real-time PCR. J Clin Microbiol. 2002;40(10):3666-3670. https://doi. org/10.1128/JCM.40.10.3666-3670.2002

23. Nix WA, Oberste MS, Pallansch MA. Sensitive, seminested PCR amplification of VP1 sequences for direct identification of all enterovirus serotypes from original clinical specimens. J Clin Microbiol. 2006;44(8):2698-2704. https://doi.org/ 10.1128/JCM.00542-06

24. Cohen AL, Hellferscee $O$, Pretorius $M$, et al. Epidemiology of influenza virus types and subtypes in South Africa, 2009-2012. Emerg Infect Dis. 2014:20(7):1162-1169. https://doi.org/10.3201/eid2007.131869

25. Benschop K, Minnaar R, Koen G, et al. Detection of human enterovirus and human parechovirus ( $\mathrm{HPeV}$ ) genotypes from clinical stool samples: Polymerase chain reaction and direct molecular typing, culture characteristics, and serotyping. Diagn Microbiol Infect Dis. 2010;68(2):166-173. https://doi.org/10.1016/j. diagmicrobio.2010.05.016
26. Tryfonos C, Richter J, Koptides D, Yiangou M, Christodoulou CG. Molecular typing and epidemiology of enteroviruses in Cyprus, 2003-2007. J Med Microbiol. 2011;60(Pt 10):1433-1440. https://doi.org/10.1099/jmm.0.029892-0

27. Tan CY, Ninove L, Gaudart J, Nougairede A, Zandotti C, Thirion-Perrier L, et al. A retrospective overview of enterovirus infection diagnosis and molecula epidemiology in the public hospitals of Marseille, France (1985-2005). PLoS One. 2011;6(3):e18022. https://doi.org/10.1371/journal.pone.0018022

28. Hsu CH, Lu CY, Shao PL, et al. Epidemiologic and clinical features of non-polio enteroviral infections in northern Taiwan in 2008. J Microbiol Immunol Infect. 2011;44(4):265-273. https://doi.org/10.1016/j.jmii.2011.01.029

29. Apostol LN, Suzuki A, Bautista A, et al. Detection of non-polio enteroviruses from 17 years of virological surveillance of acute flaccid paralysis in the Philippines. J Med Virol. 2012;84(4):624-631. https://doi.org/10.1002/jmv.23242

30. Odoom JK, Obodai E, Barnor JS, Ashun M, Arthur-Quarm J, Osei-Kwasi M. Human enteroviruses isolated during acute flaccid paralysis surveillance in Ghana: Implications for the post eradication era. Pan Afr Med J. 2012;12:74.

31. Oyero OG, Adu FD. Non-polio enteroviruses serotypes circulating in Nigeria. Afr J Med Med Sci. 2010;39(Suppl):201-208.

32. Smura $T$, Blomqvist $\mathrm{S}$, Vuorinen $\mathrm{T}$, et al. The evolution of $\mathrm{Vp} 1$ gene in enterovirus $\mathrm{C}$ species sub-group that contains types CVA-21, CVA-24, EV-C95, EV-C96 and EVspecies sub-group that contains types CVA-21, CVA-24, EV-C95, EV-C96 and EV-
C99. PLoS One. 2014;9(4):e93737. https://doi.org/10.1371/journal.pone.0093737

33. Tao Z, Yuan $Q$, Lin X, et al. Molecular characterization of enteroviruses including a new type EV-C99 isolated from Xinjiang students in Shandong, China in 2011. Sci Rep. 2014;4:6564. https://doi.org/10.1038/srep06564

34. Liu CC, Tseng HW, Wang SM, Wang JR, Su IJ. An outbreak of enterovirus 71 infection in Taiwan, 1998: Epidemiologic and clinical manifestations. J Clin Virol. 2000;17(1):23-30. https://doi.org/10.1016/S1386-6532(00)00068-8

35. Ortner B, Huang CW, Schmid D, et al. Epidemiology of enterovirus types causing neurological disease in Austria 1999-2007: Detection of clusters of echovirus 30 and enterovirus 71 and analysis of prevalent genotypes. J Med Virol. 2009;81(2):317-324. https://doi.org/10.1002/jmv.21374

36. Centre for Disease Control WHO. Enterovirus surveillance guidelines. Copenhagen: World Health Organisation Regional Office for Europe; 2015.

37. Trallero G, Avellon A, Otero A, et al. Enteroviruses in Spain over the decade 1998-2007: Virological and epidemiological studies. J Clin Virol. 2010;47(2): 170-176. https://doi.org/10.1016/j.jcv.2009.11.013

38. Oberste MS, Maher K, Kennett ML, Campbell JJ, Carpenter MS, Schnurr D, et al. Molecular epidemiology and genetic diversity of echovirus type 30 (E30): Genotypes correlate with temporal dynamics of E30 isolation. J Clin Microbiol. 1999;37(12):3928-3933.

39. Suresh S, Forgie S, Robinson J. Non-polio enterovirus detection with acute flaccid paralysis: A systematic review. J Med Virol. 2018;90(1):3-7. https://doi.org/10.1002/ jmv.24933

40. Tanel RE, Kao SY, Niemiec TM, et al. Prospective comparison of culture vs genome detection for diagnosis of enteroviral meningitis in childhood. Arch Pediatr Adolesc Med.1996;150(9):919-924.https://doi.org/10.1001/archpedi.1996.02170340033006

41. Grandien M, Fosgren M, Ehrnst A. Enteroviruses and reoviruses. 6th ed Association APH, editor. Washington, DC: American Public Health Association, 1989; p. 513-569.

42. Bal A, Schuffenecker I, Casalegno JS, et al. Enterovirus D68 nosocomial outbreak in elderly people, France, 2014. Clin Microbiol Infect. 2015;21(8):e61-e62. https://doi.org/10.1016/j.cmi.2015.05.008

43. Peci A, Winter AL, Warshawsky B, et al. Epidemiology of enterovirus D68 in Ontario. PLoS One. 2015;10(11):e0142841. https://doi.org/10.1371/journal.pone.0142841

44. Jiao MMA, Apostol LN, De Quiroz-Castro M, et al. Non-polio enteroviruses among healthy children in the Philippines. BMC Public Health. 2020;20(1):167. https:// doi.org/10.1186/s12889-020-8284-x 